\title{
Association Between Microalbuminuria and Pulse Pressure Among Patients with Isolated Systolic and Diastolic Hypertension
}

\author{
(1) Şenol Coşkun, (1) Jale Cordan, (1) Ertuğrul Mehmetoğlu, (1) Saim Sağ, (1) Dilek Yeşilbursa, \\ (1) Osman Akın Serdar, (1) Sümeyye Güllülü, (1) Ali Aydınlar
}

Bursa Uludağ University Faculty of Medicine, Department of Cardiology, Bursa, Turkey

\begin{abstract}
Objectives: The aim of this study is to examine the prevalence and predictors of microalbuminuria and to investigate the association between microalbuminuria and pulse pressure among patients with isolated systolic and diastolic hypertension (DH).

Materials and Methods: In this cross-sectional study, patients with DH and isolated systolic hypertension (ISH) were included. Data including patient age, sex, duration of hypertension (HT), comorbidities, and drugs were recorded. Blood analysis of serum total cholesterol, high D-density lipoprotein (HDL) cholesterol, triglyceride, urea, creatinine, and fasting blood glucose, urinalysis, electrocardiography (ECG), transthoracic echocardiography, exercise stress test (EST), and coronary angiography (if the patient had a positive EST result) were performed.

Results: Overall, we included 183 patients (58.5\% female) with HT. The patients with ISH had significantly higher pulse pressure, left ventricular (LV) mass, and LV mass index, but had a shorter duration of HT and lower diastolic blood pressure than those with DH. All patients were in sinus rhythm, and there were no ischemic ECG changes. Fifty-
\end{abstract}

Address for Correspondence: Şenol Coşkun, Bursa Uludă̆ University Faculty of Medicine, Department of Cardiology, Bursa, Turkey e-mail: acibademcardiology16@gmail.com ORCID: orcid.org/0000-0001-5804-0062

Received: 18.10.2020 Accepted: 04.12.2021

Cite this article as: Coşkun Ş, Cordan J, Mehmetoğlu E, Sağ S, Yeşilbursa D, Serdar OA, Güllülü S, Aydınlar A. Association Between Microalbuminuria and Pulse Pressure Among Patients with Isolated Systolic and Diastolic Hypertension.

EJCM 2021;9(1):39-48.

DOI: 10.32596/ejcm.galenos.2020-10-056

${ }^{\circ}$ Copyright 2021 by Heart and Health Foundation of Turkey (TÜSAV) / E Journal of Cardiovascular Medicine published by Galenos Publishing House. 


\begin{abstract}
nine (32.2\%) patients had microalbuminuria (MA) ( $\geq 20 \mu \mathrm{g} / \mathrm{min}$ ). LV mass, left atrial diameter, E/A ratio, systolic blood pressure (SBP), pulse pressure, and frequency of LVH were significantly higher in patients with MA than in patients without MA. The prevalence of angiographically proven atherosclerotic heart disease was similar in patients with and without microalbuminuria. Multivariate binary logistic regression analysis revealed that only systolic pressure and left atrial diameter were independent associates of microalbuminuria. Each $1 \mathrm{mmHg}$ increase in SBP was associated with a $3 \%$ increase in the risk of having microalbuminuria $(\mathrm{p}=0.04)$. In addition, each $1 \mathrm{~mm}$ increase in the diameter of the left atrium increased the risk of having microalbuminuria by $20 \%(\mathrm{p}=0.02)$.
\end{abstract}

Conclusion: This study demonstrated that microalbuminuria in hypertensive patients was independently associated with left atrial size and systolic blood pressure, but not with pulse pressure.

Keywords: Hypertension, pulse pressure, microalbuminuria, cardiac disease, isolated systolic hypertension, diastolic hypertension

\section{Introduction}

Microalbuminuria (MA) is defined as the presence of 30-300 $\mathrm{mg}$ of albumin in a 24-hour collected urine sample (or $20-200 \mathrm{mcg} / \mathrm{min})^{(1)}$. MA occurs as a result of disruption in the glomerular filtration barrier. Major causes of MA include, but not limited to, diabetes mellitus (DM), hypertension, and renal disease. In addition, diurnal variation and a number of functional causes of MA have been reported ${ }^{(2)}$.

MA is common even in the general population. Several epidemiologic studies reported the prevalence of MA in the healthy general population ranging between $2.2 \%$ and $11.8 \%{ }^{(3)}$.

Mogensen, who was the pioneer reporting the significance of albuminuria in cardiorenal disease, reported first that the presence of MA was predictive of overt proteinuria as well as cardiovascular mortality in type 2 diabetic patients ${ }^{(4)}$. After this first report, it was elucidated that there was a linear relationship between MA level and cardiovascular risk. Even albuminuria levels below the MA lower threshold were associated with increased cardiovascular risk in the Framingham Offspring Study ${ }^{(5)}$.

Hypertension is one of the major causes of MA, which is considered as a target organ damage of high blood pressure $^{(6)}$. It has been reported that MA is found in up to $15 \%$ of patients with primary hypertension ${ }^{(7)}$. On the other hand, some other studies reported an exceptionally high prevalence of MA in hypertensive patients reaching up to $50 \%{ }^{(8,9)}$.

Several factors may account for the great variability of MA prevalence among hypertensive patients. First, higher blood pressure levels are associated with higher degrees of MA. Second, factors such as exercise, fever, heart failure, and urinary tract infection may lead to temporary albumin excretion with urine. Third, some factors related to high blood pressure might be at work. For instance, different types of blood pressure patterns such as nocturnal blood pressure elevation, masked hypertension and white-coat hypertension are independently associated with MA development ${ }^{(10,11)}$. In addition, subsequent studies also showed an association between systolic (but not diastolic) blood pressure and pulse pressure with the development of $\mathrm{MA}^{(12-15)}$. Considering the magnitude of the problem, available studies investigating the relationship between blood pressure patterns and MA seem inadequate. In another population-based study, pulse pressure was reported to affect MA development in an ethnicitydependent manner ${ }^{(16)}$. Thus, further studies in different

\title{
Coşkun et al. Microalbuminuria and Hypertension
}


ethnic groups seem necessary to better understand the relationship between pulse pressure and MA.

Due to the high prevalence of MA in the general population and hypertensive patients and its unequivocal causal association with cardiovascular and renal disease, we aimed to study the prevalence and predictors of MA in hypertensive patients. We exerted special attention on the relationship between pulse pressure, systolic and diastolic hypertension (DH) as well as cardiovascular disease in a Turkish hypertensive patient cohort.

\section{Materials and Methods}

\section{Design, Setting and Population}

In this cross-sectional study, patients with $\mathrm{DH}$ and isolated systolic hypertension (ISH) were included. The study was approved by the ethics committee of Uludag University Medical Faculty (2003-18/36). Written informed consent forms were signed by each study participant.

Data including patient age, sex, duration of HT, comorbidities, and drugs were recorded for each participant. Inclusion criteria were as follows: having DH or ISH, having serum urea and creatinine levels in normal limits for age and sex, and normal urine microscopy.

Exclusion criteria were the presence of severe valvular heart disease, angina pectoris, findings of previous myocardial infarction in electrocardiography, coronary artery disease (CAD), urinary tract infection, fasting blood glucose $>110 \mathrm{mg} / \mathrm{dL}$, having with pacing rhythm and atrial fibrillation, segmentary wall motion abnormalities, thyroid disease, anemia, liver disease, chronic obstructive pulmonary disease and secondary hypertension.

\section{Laboratory Evaluations}

Blood analysis of serum total cholesterol (TC), high density lipoprotein (HDL) cholesterol, triglyceride (TG), urea, creatinine, and fasting blood glucose and urinalysis were performed after 12 hours of fasting. Low density lipoprotein (LDL) cholesterol was calculated according to the Friedewald formula as follows: LDL $(\mathrm{mg} / \mathrm{dL})=\mathrm{TC}$ $(\mathrm{mg} / \mathrm{dL})$ - HDL $(\mathrm{mg} / \mathrm{dL})-\mathrm{TG}(\mathrm{mg} / \mathrm{dL}) / 5^{(17)}$.
MA level was measured in 24-hour urine by an immunometric assay with fluorescence detection on the DPC IMMULITE 2000 analyzer (Euro/DPC Ltd, Llanberis, UK). Urinary albumin concentration of $\geq 20$ $\mu \mathrm{g} /$ minute (approximates to $30 \mathrm{mg}$ /day) was considered as MA.

\section{Anthropometric and Blood Pressure Measurements}

Patient height and weight were measured. Body mass index was calculated by dividing weight in kilograms by the square of height in meters ${ }^{(18)}$.

Blood pressure (BP) was measured three times with two-minute intervals, from the right arm with a mercury manometer based on Korotkoff sounds, sitting upright position, after a rest of at least 10 minutes. The arithmetic mean of the three BP values was calculated for each patient as the final BP measurement.

ISH was defined when systolic BP was $\geq 140 \mathrm{mmHg}$ and diastolic BP was $<90 \mathrm{mmHg}^{(19)}$. $\mathrm{DH}$ was diagnosed according to the Joint National Committee (JNC7) guideline (systolic BP less than $140 \mathrm{mmHg}$ and a diastolic $\mathrm{BP}$ of at least $90 \mathrm{mmHg})^{(19)}$. Pulse pressure was calculated by subtracting the diastolic BP value from systolic BP value. All anthropometric and BP measurements were performed by the same investigator.

\section{Cardiac Evaluation}

Standard 12-lead electrocardiography (ECG), transthoracic echocardiography, and exercise stress tests (EST) were performed in all patients. If the patient had a positive EST result, coronary angiography was performed additionally.

Two-dimensional, M-mode, and Doppler echocardiography was performed using the SONOS 2000 device (Hewlett-Packard Medical Systems, Andover, MA, USA) in accordance with the recommendations of the American Society of Echocardiography ${ }^{(20)}$. Echocardiography was performed while the participant was lying on the left side. Doppler measurements were carried out during expiration. All measurements were 
taken by the same experienced echocardiography operator who was blinded to the status of the patient. E wave, A wave, and E/A ratio were measured by taking pulsed Doppler records of the mitral valve along with ECG recording. Left ventricular (LV) systolic and diastolic diameter, interventricular septal (IVS), and LV posterior wall thickness (PWT) were measured. Interventricular septal (IVS) and LV PWT, and end-diastolic diameter (EDD) were calculated with the Devereux formula ${ }^{(21)}$. LV mass index (LVMI) was computed using the following equation: $\mathrm{LVMI}=\mathrm{LV}$ mass/body surface area. A patient was considered to have LV hypertrophy (LVH) if the LV mass index was greater than $134 \mathrm{~g} / \mathrm{m}^{2}$ in men and greater than $110 \mathrm{~g} / \mathrm{m}^{2}$ in women ${ }^{(22,23)}$.

The EST was performed with the standard Bruce protocol $^{(24)}$. Drugs that could interfere with the test results, such as beta-blockers, calcium channel blockers, digoxin, etc., were discontinued twice the drug half-life before the test. The test was terminated in case of obtaining the predetermined heart rate (220-age), development of severe shortness of breath, chest pain, weakness, ST depression ( $>3 \mathrm{~mm})$, ST-elevation $(>2 \mathrm{~mm})$, ventricular or supraventricular tachyarrhythmias, left bundle branch block, second or third-degree block, decrease (more than $20 \mathrm{mmHg}$ ) or increase (more than $250 \mathrm{mmHg}$ ) in systolic blood pressure (SBP). The result was interpreted as positive in terms of ischemia in the presence of ST elevation ( $>1 \mathrm{~mm}$, in derivation that without $\mathrm{Q}$ wave) or downsloping ST depression in at least two successive leads after 80 milliseconds from the $\mathrm{J}$ point, $20 \mathrm{mmHg}$ decrease in SBP, occurrence of angina pectoris, and angina equivalent shortness of breath.

Coronary angiography was performed in patients whose EST result was positive for ischemia by using the femoral artery with the Judkin's technique (Philips DCI device $)^{(25)}$. The coronary angiograms were interpreted by experienced invasive cardiologists. Atherosclerotic plaques in the epicardial coronary arteries (greater than $30 \%$ in lumen narrowing) were accepted as $\mathrm{CAD}^{(26)}$.

\section{Statistical Analysis}

The Kolmogorov-Smirnov test was used to check the normality assumptions of the data. Normally distributed variables were presented as a mean \pm standard deviation, whereas non-normally distributed variables were given as median and min-max. The Independent samples t-test or the Mann-Whitney-U test were used for numerical variables, and chi-square was employed for categorical variables. Binary logistic regression analysis was performed to determine independent associates of MA.

SPSS 10.0 software package (IBM, Armonk, NY, USA) was used to analyze data of the study. A p-value $<0.05$ was accepted as statistically significant.

\section{Results}

Overall, we included 183 patients (58.5\% female) with hypertension. The mean age of the entire study cohort was $55.1 \pm 9.2$ years (range: $31-80$ years). The median length of duration of hypertension was 4 years (ranging from 1 to 20 years). Clinicodemographic characteristics and laboratory values of the entire study cohort are shown in Table 1 .

When the patients who had DH and ISL were compared, it was observed that patients with ISL had significantly higher pulse pressure, LV mass, and LV mass index, but had a shorter duration of HT and lower diastolic BP. Moreover, patients with ISL were significantly older than the patients with DH (Table 2). The prevalence of angiographically proven atherosclerotic heart disease was similar in patients with and without MA.

We divided the study population into two groups according to their MA levels. While patients with $M A \geq 20$ $\mu \mathrm{g} / \mathrm{min}$ were considered MA positive group, those with MA $<20 \mu \mathrm{g} / \mathrm{min}$ were defined as MA negative group. Fifty-nine (32.2\%) patients were MA positive. The resting ECG parameters were compared in patients with and without MA. All patients were in sinus rhythm, and there were no ischemic ECG changes. Patients with MA were significantly older than patients without MA. Besides, LV mass, LVMI, left atrial diameter, E/A ratio, SBP, pulse 
Table 1. Clinicodemographic characteristics and laboratory values of the entire study cohort

\section{Parameters}

Age, (years)

\section{Gender}

Female/male

BMI, $\left(\mathrm{kg} / \mathrm{m}^{2}\right)$

Comorbidities, $\mathbf{n}(\%)$

Coronary artery disease (family history)

Duration of hypertension, (years)

Dyslipidemia

Microalbuminuria

Smoking

Antihypertensive medications, $\mathrm{n}(\%)$

No medication

ACE inhibitors

Beta-blockers

Calcium channel blockers

Multi-drug

Laboratory parameters

Fasting blood glucose, (mg/dL)

Total cholesterol, (mg/dL)

LDL-cholesterol, (mg/dL)

HDL-cholesterol, (mg/dL)

Triglycerides, $(\mathrm{mg} / \mathrm{dL})$

Microalbuminuria, ( $\mu \mathrm{g} / \mathrm{min})$

Blood pressure measurements

Systolic blood pressure, $(\mathrm{mmHg})$

Diastolic blood pressure, $(\mathrm{mmHg})$

Pulse pressure, $(\mathrm{mmHg})$

BMI: Body mass index, ACE: Angiotensin-converting enzyme, LDL: Low density lipoprotein, HDL: High density lipoprotein, max: Maximum, min: Minimum $S D$ : Standard deviation, $n$ : Number

\section{Values \\ (mean \pm SD or median and min-max)}

$55 \pm 9.2$

107 (58.5\%)/76 (41.5\%)

$27.9 \pm 4.1$

$26.2 \%$

4 (max: 20, min: 1)

$39.3 \%$

$59(32.2 \%)$

$20.2 \%$

$27(14.9 \%)$

$15 \%$

$29 \%$

$15 \%$

$26.1 \%$

$81 \pm 11$

$206 \pm 40$

$128 \pm 37$

$46 \pm 7$

133 (max: 386, min: 35)

13 (max: 179, $\min : 1.2$ )

$152.2 \pm 19.2$

$91.1 \pm 9.3$

$61.1 \pm 17$ pressure, and frequency of LVH were significantly higher in patients with MA than those without MA (Table 3).

Multivariate binary logistic regression analysis revealed that only systolic pressure and left atrial diameter were independent associates of MA. Each $1 \mathrm{mmHg}$ increase in SBP was associated with a 3\% increase in the risk of having MA ( $\mathrm{p}=0.04)$. In addition, each $1 \mathrm{~mm}$ increase in the diameter of the left atrium increased the risk of having MA by $20 \%(\mathrm{p}=0.02)$ (Table 4$)$.

\section{Discussion}

The notable findings of the present study were as follows: (i) there was not a difference between the patients with diastolic and ISH in terms of rate of MA. (ii) SBP and pulse pressure were statistically significantly higher among patients who had MA compared to patients without MA. (iii) Although there was no difference regarding the rate of $\mathrm{CAD}$, patients with MA had a significantly higher LV mass and LV mass index relative to patients without MA. (iv) Multivariate logistic regression analysis 
Table 2. Comparison of demographic characteristics, clinical and laboratory data of patients with diastolic hypertension and isolated systolic hypertension

\begin{tabular}{|c|c|c|c|}
\hline & $\begin{array}{l}\text { Diastolic hypertension } \\
(n=124)\end{array}$ & $\begin{array}{l}\text { Isolated systolic } \\
\text { hypertension }(n=59)\end{array}$ & p-value \\
\hline Age, (years) & $51.5 \pm 7.9$ & $62.6 \pm 6.9$ & 0.001 \\
\hline \multicolumn{4}{|l|}{ Gender } \\
\hline Female/male & $76 / 48$ & $31 / 28$ & NS \\
\hline BMI, $\left(\mathrm{kg} / \mathrm{m}^{2}\right)$ & $28.1 \pm 4.2$ & $27.5 \pm 3.7$ & NS \\
\hline \multicolumn{4}{|l|}{ Comorbidities, n (\%) } \\
\hline Duration of hypertension & 5 (max: $10 \min : 3)$ & 3.5 (max: 20 min: 1) & 0.007 \\
\hline Microalbuminuria positivity & $28 \%$ & $40.7 \%$ & NS \\
\hline \multicolumn{4}{|c|}{ Echocardiographic left ventricular measurements } \\
\hline Left ventricular mass, (gram) & $197 \pm 55.3$ & $246.9 \pm 75$ & $<0.001$ \\
\hline Left ventricular mass index, (gram $\left./ \mathrm{m}^{2}\right)$ & $106 \pm 27.5$ & $134.7 \pm 40$ & $<0.001$ \\
\hline \multicolumn{4}{|l|}{ Laboratory parameters } \\
\hline Fasting blood glucose, (mg/dL) & $87 \pm 11$ & $91 \pm 10$ & NS \\
\hline Total cholesterol, (mg/dL) & $205 \pm 39$ & $207 \pm 43$ & NS \\
\hline LDL-cholesterol, (mg/dL) & $127 \pm 38$ & $131 \pm 35$ & NS \\
\hline HDL-cholesterol, (mg/dL) & $46.8 \pm 9.6$ & $46.6 \pm 9.7$ & NS \\
\hline Triglycerides, (mg/dL) & 132 & 137 & NS \\
\hline Microalbuminuria, ( $\mu \mathrm{g} / \mathrm{min})$ & 13.1 & 12.8 & NS \\
\hline \multicolumn{4}{|l|}{ Blood pressure measurements } \\
\hline Systolic blood pressure, $(\mathrm{mmHg})$ & $149.1 \pm 18.1$ & $159.1 \pm 18.1$ & 0.001 \\
\hline Diastolic blood pressure, $(\mathrm{mmHg})$ & $93 \pm 10.3$ & $83.6 \pm 5.5$ & $<0.001$ \\
\hline Pulse pressure, $(\mathrm{mmHg})$ & $56.1 \pm 14$ & $71.8 \pm 18$ & $<0.001$ \\
\hline
\end{tabular}

revealed that independent predictors of MA were SBP and left atrial diameter.

The causal association between elevated BP and the development of MA has been firmly established ${ }^{(27,28)}$. Moreover, it has been demonstrated that not only the level of BP but also several characteristics of BP elevation such as systolic and diastolic components, pulse pressure, nondipping pattern (nocturnal hypertension) had an impact in this respect ${ }^{(10,11,29)}$. However, studies exploring the relationship between $\mathrm{BP}$ components such as pulse pressure and SBP are limited ${ }^{(12,14,15,30,31)}$. Several hypotheses have been put forward to explain the effect of systolic and pulse pressures on MA development. Actually, SBP is reflected in the glomerulus and associated with intraglomerular HT and consequent glomerular injury ${ }^{(32,33)}$. A study recruited
1,858 treatment-naïve hypertensive patients and they found SBP [odds ratio (OR): 1.010, confidence interval $(\mathrm{CI})$ : $1.005-1.016, \mathrm{p}<0.001]$ and pulse pressure $(\mathrm{OR}$ : 1.009, 95\% CI: 1.003-1.015, $\mathrm{p}=0.003$ ) as independent predictors of $\mathrm{MA}^{(27)}$.

In our study, the only independent associates of MA were left atrial diameter and SBP. In contrast to previous studies, pulse pressure was not found as an independent predictor of MA in hypertensive patients. Since our patients were not treatment-naïve, this might have led to the negative finding of pulse pressure and MA interaction. On the other hand, each $1 \mathrm{mmHg}$ increase in SBP was associated with a $3 \%$ increased risk of MA.

MA is long established as an independent cardiovascular risk factor ${ }^{(33)}$. This effect is independent of accompanying

\section{Coşkun et al. Microalbuminuria and Hypertension}


Table 3. Comparison of demographic characteristics, clinical and laboratory data of patients with and without microalbuminuria

\begin{tabular}{|c|c|c|c|}
\hline & $\begin{array}{l}\text { Patients with MA } \\
\text { (MA } \geq 20 \mu \mathrm{g} / \mathrm{min} \text { ) } \\
\text { ( } \mathrm{n}=59)\end{array}$ & $\begin{array}{l}\text { Patients without MA } \\
\text { (MA }<20 \mu \mathrm{g} / \mathrm{min}) \\
(\mathrm{n}=124)\end{array}$ & p-value \\
\hline Age, (years) & $57.2 \pm 9.9$ & $54.3 \pm 8.8$ & 0.045 \\
\hline Female/male & $32 / 27$ & $75 / 49$ & NS \\
\hline BMI, $\left(\mathbf{k g} / \mathrm{m}^{2}\right)$ & $27.4 \pm 4.8$ & $28.2 \pm 3.7$ & NS \\
\hline Coronary artery disease (family history) & $32 \%$ & $23.4 \%$ & NS \\
\hline Duration of hypertension, (years) & 5.7 (max: 19, $\min : 1)$ & 5.3 (max: 20, $\min : 1)$ & NS \\
\hline Dyslipidemia & $30.5 \%$ & $43.5 \%$ & NS \\
\hline Smoking & $13.6 \%$ & $23.6 \%$ & NS \\
\hline \multicolumn{4}{|l|}{ Cardiac measurements } \\
\hline LVMI (in ECHO), $\left(\mathrm{g} / \mathrm{m}^{2}\right)$ & $125.6 \pm 34.6$ & $111 \pm 34.1$ & $<0.001$ \\
\hline Left atrial diameter, $(\mathrm{mm})$ & $39.1 \pm 3.26$ & $37.3 \pm 3.2$ & $<0.001$ \\
\hline E wave & $0.69 \pm 0.19$ & $0.68 \pm 0.17$ & NS \\
\hline A wave & $0.73 \pm 0.18$ & $0.75 \pm 0.16$ & NS \\
\hline E/A ratio & $1.03 \pm 0.46$ & $0.94 \pm 0.33$ & 0.014 \\
\hline E wave DT, (milliseconds) & $178.3 \pm 50.8$ & $182.5 \pm 47.5$ & NS \\
\hline IVRT, (milliseconds) & $91.2 \pm 28.7$ & $94.1 \pm 21.4$ & NS \\
\hline Positive stress test result, (\%) & $9(15.3 \%)$ & $13(10.5 \%)$ & NS \\
\hline Coronary artery disease, $(\%)$ & $9(15.3 \%)$ & $8(6.5 \%)$ & NS \\
\hline Microalbuminuria, ( $\mu \mathrm{g} / \mathrm{min})$ & 25.8 & 10.6 & $<0.001$ \\
\hline \multicolumn{4}{|l|}{ Blood pressure measurements } \\
\hline Systolic blood pressure, $(\mathrm{mmHg})$ & $159.5 \pm 19.1$ & $148.9 \pm 18.5$ & $<0.001$ \\
\hline Diastolic blood pressure, $(\mathrm{mmHg})$ & $91.2 \pm 11.6$ & $89 \pm 9.2$ & NS \\
\hline Pulse pressure, $(\mathrm{mmHg})$ & $66.1 \pm 17.1$ & $58.8 \pm 17.2$ & 0.008 \\
\hline
\end{tabular}

hypertension or DM, too. Some hypotheses regarding this independent and strong relationship point to the fact that MA is a marker of generalized endothelial dysfunction, which also underpins the development of atherosclerotic heart disease ${ }^{(34-36)}$. Wider pulse pressure operates as a marker of the pulsatile hemodynamic load and conduit vessel stiffness. Hence pulse pressure seems as a significant cardiovascular risk factor ${ }^{(15)}$. Thus, wider pulse pressure 
Table 4. Multivariate logistic regression analysis to determine the independent associates of microalbuminuria

\begin{tabular}{|l|l|l|l|}
\hline \multicolumn{4}{|c|}{ Multivariate LR } \\
\hline Variables & OR & $\mathbf{9 5 \%} \mathbf{C l}$ & p-value \\
\hline Systolic blood pressure & 1.03 & $1.009-1.046$ & 0.04 \\
\hline Left atrial diameter & 1.202 & $1.073-1.347$ & 0.02 \\
\hline OR: Odds ratio, Cl: Confidence interval, LR: Logistic regression
\end{tabular}

seems like a plausible common risk factor impacting both MA and atherosclerotic heart disease. Recently, pulse pressure has been linked to diabetes inflammation and obesity, all of which are associated with MA. Thus, pulse pressure might be related to the development of MA not only through high BP but also with diabetes, inflammation and obesity ${ }^{(37)}$.

Our results revealed that the prevalence of atherosclerotic disease that was demonstrated with coronary angiography among hypertensive patients with MA was no different from those who did not have MA. On the other hand, microalbuminuric patients had significantly higher rates of LV mass, LV mass index, and left atrial diameter relative to non-microalbuminuric patients. The lack of difference regarding atherosclerotic disease between the groups might be due to the relatively small sample size of our study and drug use, such as statins and angiotensin-converting enzyme (ACE) inhibitors that might affect the development of manifest atherosclerotic disease.

Some limitations of the present work are as follows: First, we did not recruit treatment-naïve patients. We are not sure that patients on statin and ACE inhibitor treatments were matched between the microalbuminuric and nonmicroalbuminuric patients. Second, we did not employ ambulatory BP measurement, which was shown to be a better predictor of cardiovascular morbidity and mortality. Instead, we relied on the office measurement, which may be suffered from the white coat effect. Third, our sample size might not be sufficient to elucidate subtle differences between the MA groups.
Despite its limitations, we think that current work makes important contributions to the literature. We evaluated the association between MA and BP components in the context of cardiovascular disease. We evaluated the cardiac function of the study participants in detail with echocardiography, ESTs, and angiography, when needed. Thus, we think that our results portrayed a more realworld situation in which the association of MA and BP was investigated.

\section{Conclusion}

This present study demonstrated that MA in prevalent hypertensive patients was independently associated with left atrial size and systolic BP, but not with pulse pressure. Moreover, the frequency of proven atherosclerotic heart disease was not different between the microalbuminuric and non-microalbuminuric hypertensive patients. It seems there is an exact need to conduct more studies in this field.

\section{Ethics}

Ethics Committee Approval: The study was approved by the Ethics Committee of Uludağ University Medical Faculty (2003-18/36).

Informed Consent: Written informed consent forms were signed by each study participant.

Peer-review: Externally peer-reviewed.

\section{Authorship Contributions}

Conception and design: Ş.C., J.C., D.Y., Acquisition of data: E.M., S.S., Drafting of the manuscript: Ş.C., S.S., Critical revision of the manuscript for important intellectual content: J.C., D.Y., O.A.S., Statistical analysis: Ş.C., Administrative, technical or material support: D.Y., Supervision: J.C., D.Y., O.A.S., S.G., A.A.

Conflict of Interest: The authors declared no conflict of interest.

Financial Disclosure: The authors declared that this study received no financial support. 


\section{References}

1. Basi S, Fesler P, Mimran A, Lewis JB. Microalbuminuria in type 2 diabetes and hypertension: a marker, treatment target, or innocent bystander? Diabetes Care 2008;31:194-201.

2. Segura J, Ruilope LM, Rodicio JL. Microalbuminuria. Clin Exp Hypertens 2004;26:701-7.

3. Yuyun MF, Khaw KT, Luben R, et al. Microalbuminuria, cardiovascular risk factors and cardiovascular morbidity in a British population: the EPIC-Norfolk population-based study. Eur J Cardiovasc Prev Rehabil 2004;11:207-13.

4. Mogensen CE. Microalbuminuria predicts clinical proteinuria and early mortality in maturity-onset diabetes. N Engl J Med 1984;310:356-60.

5. Arnlöv J, Evans JC, Meigs JB, et al. Low-grade albuminuria and incidence of cardiovascular disease events in nonhypertensive and nondiabetic individuals: the Framingham Heart Study. Circulation 2005;112:969-75.

6. Reboldi G, Gentile G, Angeli F, Verdecchia P. Microalbuminuria and hypertension. Minerva Med 2005;96:261-75.

7. Jones CA, Francis ME, Eberhardt MS, et al. Microalbuminuria in the US population: third National Health and Nutrition Examination Survey. Am J Kidney Dis 2002;39:445-59.

8. Böhm M, Thoenes M, Danchin N, Bramlage P, La Puerta P, Volpe M. Association of cardiovascular risk factors with microalbuminuria in hypertensive individuals: the i-SEARCH global study. J Hypertens $2007 ; 25: 2317-24$

9. Aggarwal HK, Jain D, Mor S, Yadav RK, Jain P. Prevalence and Clinical Correlates of Microalbuminuria in Patients with Essential Hypertension - A Tertiary Care Center Cross Sectional Study. J Assoc Physicians India 2018;66:30-4

10. Mateo-Gavira I, Vílchez-López FJ, García-Palacios MV, Carral-San Laureano F, Jiménez-Carmona S, Aguilar-Diosdado M. Nocturnal blood pressure is associated with the progression of microvascular complications and hypertension in patients with type 1 diabetes mellitus. J Diabetes Complications 2016;30:1326-32.

11. Hata J, Fukuhara M, Sakata S, et al. White-coat and masked hypertension are associated with albuminuria in a general population: the Hisayama Study. Hypertens Res 2017;40:937-43.

12. Cirillo M, Stellato D, Laurenzi M, Panarelli W, Zanchetti A, De Santo NG (2000) Pulse pressure and isolated systolic hypertension: association with microalbuminuria. Kidney İnternational 2000;58:1211-8.

13. Xie N, Li X, Zhong Q, et al. Association of systolic blood pressure and pulse pressure with microalbuminuria in treatment-naïve hypertensive patients. Arch Med Sci 2019;15:832-6.

14. Tsakiris A, Doumas M, Lagatouras D, et al. Microalbuminuria is determined by systolic and pulse pressure over a 12-year period and related to peripheral artery disease in normotensive and hypertensive subjects: the Three Areas Study in Greece (TAS-GR). Angiology 2006;57:313-20.

15. Pedrinelli R, Dell'Omo G, Penno G, et al. Microalbuminuria and pulse pressure in hypertensive and atherosclerotic men. Hypertension 2000;35:48-54.

16. Agyemang C, van Valkengoed I, van den Born BJ, Stronks K. Prevalence of microalbuminuria and its association with pulse pressure in a multi-ethnic population in Amsterdam, the Netherlands. The SUNSET Study. Kidney Blood Press Res 2008;31:38-46.

17. Tremblay AJ, Morrissette H, Gagné JM, Bergeron J, Gagné C, Couture P. Validation of the Friedewald formula for the determination of lowdensity lipoprotein cholesterol compared with beta-quantification in a large population. Clin Biochem 2004;37:785-90.

18. Clinical Guidelines on the Identification, Evaluation, and Treatment of Overweight and Obesity in Adults--The Evidence Report. National Institutes of Health. Obes Res 1998;6:51-209.

19. Chobanian AV, Bakris GL, Black HR, et al. Seventh report of the Joint National Committee on Prevention, Detection, Evaluation, and Treatment of High Blood Pressure. Hypertension 2003;42:1206-52.

20. Mancia G, Fagard R, Narkiewicz K, et al. 2013 ESH/ESC guidelines for the management of arterial hypertension: the Task Force for the Management of Arterial Hypertension of the European Society of Hypertension (ESH) and of the European Society of Cardiology (ESC). Eur Heart J 2013;34:2159219.

21. Sahn DJ, DeMaria A, Kisslo J, Weyman A. Recommendations regarding quantitation in M-mode echocardiography: results of a survey of echocardiographic measurements. Circulation 1978;58:1072-83.

22. Jafary FH. Devereux formula for left ventricular mass--be careful to use the right units of measurement. J Am Soc Echocardiogr 2007;20:783.

23. Devereux RB, Alonso DR, Lutas EM, et al. Echocardiographic assessment of left ventricular hypertrophy: comparison to necropsy findings. Am J Cardiol 1986;57:450-8.

24. Bruce RA, Kusumi F, Hosmer D. Maximal oxygen intake and nomographic assessment of functional aerobic impairment in cardiovascular disease. Am Heart J 1973;85:546-62.

25. Page HL Jr. The Judkin's technique. Cathet Cardiovasc Diagn 1979;5:1879.

26. Arbab-Zadeh A, Fuster V. The Risk Continuum of Atherosclerosis and its Implications for Defining CHD by Coronary Angiography. J Am Coll Cardiol 2016;68:2467-78

27. Xia F, Liu G, Shi Y, Zhang Y. Impact of microalbuminuria on incident coronary heart disease, cardiovascular and all-cause mortality: a metaanalysis of prospective studies. Int J Clin Exp Med 2015;8:1-9.

28. Palatini P. Microalbuminuria in hypertension. Curr Hypertens Rep 2003;5:208-14.

29. Karadag B, Ozyigit T, Serindag Z, Ilhan A, Ozben B. Blood pressure profile is associated with microalbuminuria and retinopathy in hypertensive nondiabetic patients. Wien Klin Wochenschr 2018;130:204-10.

30. Tanaka M, Babazono T, Takeda M, Iwamoto Y. Pulse pressure and chronic kidney disease in patients with type 2 diabetes. Hypertens Res 2006;29:34552.

31. Thieme K, Oliveira-Souza M. Renal hemodynamic and morphological changes after 7 and 28 days of leptin treatment: the participation of angiotensin II via the AT1 receptor. PLoS One 2015;10:122265.

32. Kusunoki H, Taniyama Y, Rakugi H, Morishita R. Cardiac and renal protective effects of irbesartan via peroxisome proliferator-activated receptor $\gamma$-hepatocyte growth factor pathway independent of angiotensin II 
Type 1a receptor blockade in mouse model of salt-sensitive hypertension. $\mathbf{J}$ Am Heart Assoc 2013;2:000103.

33. Diercks GF, van Boven AJ, Hillege JL, de Jong PE, Rouleau JL, van Gilst WH. The importance of microalbuminuria as a cardiovascular risk indicator: A review. Can J Cardiol 2002;18:525-35.

34. Khosla N, Sarafidis PA, Bakris GL. Microalbuminuria. Clin Lab Med 2006;26:635-53.

35. Stehouwer CD, Henry RM, Dekker JM, Nijpels G, Heine RJ, Bouter LM. Microalbuminuria is associated with impaired brachial artery, flow- mediated vasodilation in elderly individuals without and with diabetes: further evidence for a link between microalbuminuria and endothelial dysfunction--the Hoorn Study. Kidney Int Suppl 2004;(92):42-4.

36. Stehouwer CD, Smulders YM. Microalbuminuria and risk for cardiovascular disease: Analysis of potential mechanisms. J Am Soc Nephrol 2006;17:2106-11.

37. Strandberg TE, Pitkala K. What is the most important component of blood pressure: systolic, diastolic or pulse pressure? Curr Opin Nephrol Hypertens 2003;12:293-7. 